\title{
Exact mathematical Formula that connect 6 dimensionless physical
} constants

\author{
Stergios Pellis ${ }^{1}$ \\ ${ }^{1}$ Affiliation not available
}

August 30, 2022

\begin{abstract}

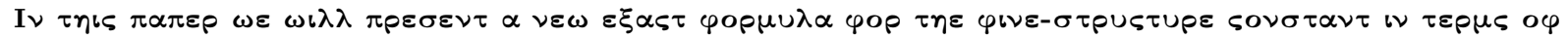

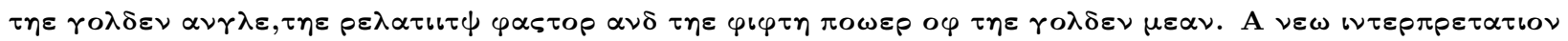

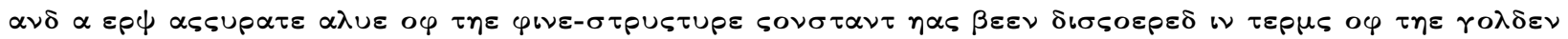

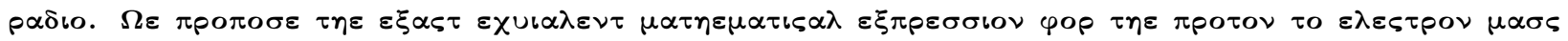

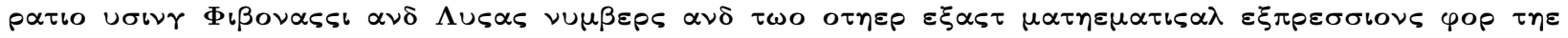

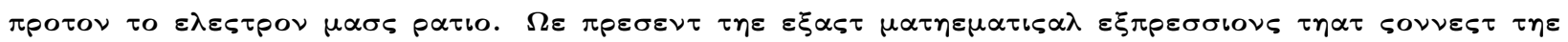

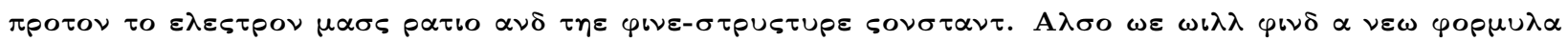

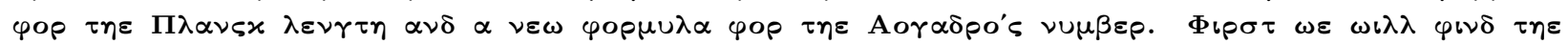

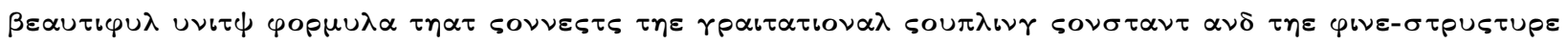

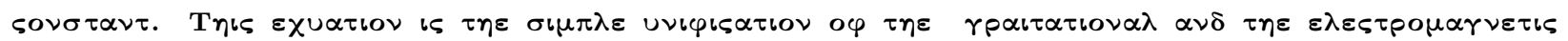

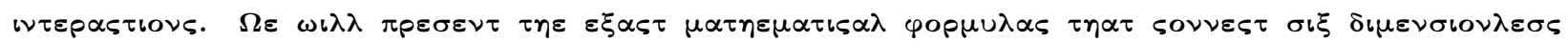

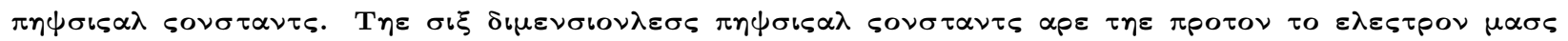

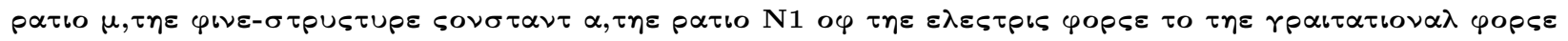

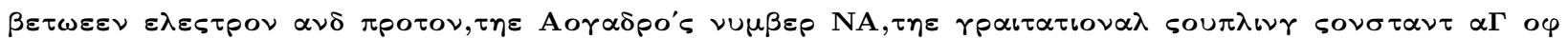

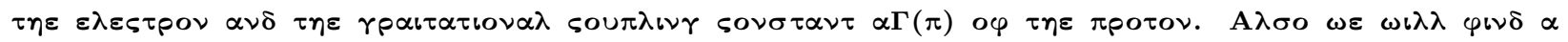
$\nu \varepsilon \omega$

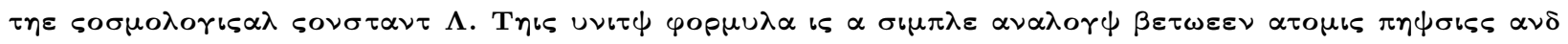

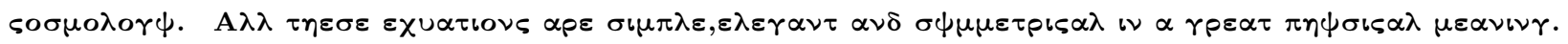




\title{
Exact mathematical formula that connect 6 dimensionless physical constants
}

\author{
Stergios Pellis \\ sterpellis@gmail.com \\ http://physiclessons.blogspot.com/ \\ Greece \\ 17 October 2.021
}

\begin{abstract}
In this paper we will present a new exact formula for the fine-structure constant in terms of the golden angle,the relativity factor and the fifth power of the golden mean. A new interpretation and a very accurate value of the fine-structure constant has been discovered in terms of the golden radio. We propose the exact equivalent mathematical expression for the proton to electron mass ratio using Fibonacci and Lucas numbers and two other exact mathematical expressions for the proton to electron mass ratio. We present the exact mathematical expressions that connect the proton to electron mass ratio and the fine-structure constant. Also we will find a new formula for the Planck length and a new formula for the Avogadro's number. First we will find the beautiful unity formula that connects the gravitational coupling constant and the fine-structure constant. This equation is the simple unification of the gravitational and the electromagnetic interactions. We will present the exact mathematical formulas that connect six dimensionless physical constants. The six dimensionless physical constants are the proton to electron mass ratio $\mu$, the fine-structure constant a,the ratio $\mathrm{N} 1$ of the electric force to the gravitational force between electron and proton, the Avogadro's number NA,the gravitational coupling constant ag of the electron and the gravitational coupling constant $\mathrm{aG}(\mathrm{p})$ of the proton. Also we will find a new formula for the gravitational constant G. Finally we will be presented the formula for the cosmological constant $\Lambda$. This unity formula is a simple analogy between atomic physics and cosmology. All these equations are simple,elegant and symmetrical in a great physical meaning.
\end{abstract}

\section{Keywords}

Fine-structure constant, Proton to electron mass ratio, Dimensionless physical constants , Gravitational constant , Avogadro's number, Gravitational constant, Ratio of the electric force to the gravitational force, Gravitational coupling constant, Cosmological constant

\section{Introduction}

In physics, a dimensionless physical constant is a physical constant that is dimensionless, a pure number having no units attached and having a numerical value that is independent of whatever system of units may be used. The term fundamental physical constant is used to refer to some universal dimensionless constants. A long-sought goal of theoretical physics is to find first principles from which all of the fundamental dimensionless constants can be calculated and compared to the measured values.

In the 1.920s and 1.930s, Arthur Eddington embarked upon extensive mathematical investigation into the relations between the fundamental quantities in basic physical theories,later used as part of his effort to construct an overarching theory unifying quantum mechanics and cosmological physics. The mathematician Simon Plouffe has made an extensive search of computer databases of mathematical formulas,seeking formulas for the mass ratios of the fundamental particles. An empirical relation between the masses of the electron,muon and tau has been discovered by physicist Yoshio Koide, but this formula remains unexplained. 
Dimensionless physical constants cannot be derived and have to be measured. Developments in physics may lead to either a reduction or an extension of their number:discovery of new particles,or new relationships between physical phenomena,would introduce new constants, while the development of a more fundamental theory might allow the derivation of several constants from a more fundamental constant. A long-sought goal of theoretical physics is to find first principles from which all of the fundamental dimensionless constants can be calculated and compared to the measured values.

The laws of physics have a set of fundamental constants,and it is generally admitted that only dimensionless combinations of constants have physical significance. These combinations include the electromagnetic and gravitational fine structure,along with the ratios of elementary particles masses. Cosmological measurements clearly depend on the values of these constants in the past and can therefore give information on their time dependence if the effects of time-varying constants can be separated from the effects of cosmological parameters.

In [7] we presented exact and approximate expressions between the Archimedes constant $n$, the golden ratio $\varphi$, the Euler's number e and the imaginary number i.

\section{Fine-structure constant}

The fine-structure constant is one of the most fundamental constants of physics. It describes the strength of the force of electromagnetism between elementary particles in what is known as the standard model of particle physics. In particular, the fine-structure constant sets the strength of electromagnetic interaction between light (photons) and charged elementary particles such as electrons and muons. The quantity a was introduced into physics by A. Sommerfeld in 1.916 and in the past has often been referred to as the Sommerfeld fine-structure constant. In order to explain the observed splitting or fine structure of the energy levels of the hydrogen atom, Sommerfeld extended the Bohr theory to include elliptical orbits and the relativistic dependence of mass on velocity.

One of the most important numbers in physics is the fine-structure constant a which defines the strength of the electro-magnetic field. It is a dimensionless number independent of how we define our units of mass,length,time or electric charge. A change in these units of measurement leaves the dimensionless constant unchanged. The number can be seen as the chance that an electron emits or absorbs a photon. It's a pure number that shapes the universe to an astonishing degree. Paul Dirac considered the origin of the number «the most fundamental unsolved problem of physics». The constant is everywhere because it characterizes the strength of the electromagnetic force affecting charged particles such as electrons and protons. Many eminent physicists and philosophers of science have pondered why $\alpha$ itself has the value that it does,because the value shows up in so many important scenarios and aspects of physics. Nobody has come up with any ideas that are even remotely convincing. A similar situation occurs with the proton-electron mass ratio $\mu$,not because of its ubiquity,but rather how chemistry can be based on two key electrically charged particles of opposite electric charge that are opposite but of seemingly identical magnitude while their masses have a ratio that is quite large yet finite. These two questions have a huge bearing on why physics and chemistry behave the way they do. The product of the two quantities appears,at least at first glance,not to be so important. The fine-structure constant a is defined as:

$$
\alpha=\frac{q_{e}^{2}}{4 \pi \varepsilon_{0} \hbar c}
$$

The 2.018 CODATA recommended value of fine-structure constant is:

$$
a=0.0072973525693(11)
$$

With standard uncertainty $0,0000000011 \times 10^{-3}$ and relative standard uncertainty $1,5 \times 10^{-10}$. For reasons of convenience, historically the value of the reciprocal of the fine-structure constant is often specified. The 2.018 CODATA recommended value of $a^{-1}$ is given by:

$$
a^{-1}=137,035999084(21)
$$


With standard uncertainty $0,000000021 \times 10^{-3}$ and relative standard uncertainty $1,5 \times 10^{-10}$. There is general agreement for the value of a,as measured by these different methods. The preferred methods in 2.019 are measurements of electron anomalous magnetic moments and of photon recoil in atom interferometry. The most precise value of a obtained experimentally (as of 2.012) is based on a measurement of $\mathrm{g}$ using a one-electron so-called "quantum cyclotron" apparatus,together with a calculation via the theory of QED that involved 12.672 tenth-order Feynman diagrams:

$$
a^{-1}=137,035999174(35)
$$

This measurement of a has a relative standard uncertainty of $2,5 \times 10^{-10}$. This value and uncertainty are about the same as the latest experimental results. Further refinement of this work were published by the end of 2.020,giving the value:

$$
a^{-1}=137,035999206(11)
$$

with a relative accuracy of 81 parts per trillion. Also the fine-structure constant is universal scaling factor:

$$
\alpha=\frac{2 \pi r_{e}}{\lambda_{e}}=\frac{\lambda_{e}}{2 \pi \alpha_{0}}=\frac{r_{e}}{l_{p l}} \frac{m_{e}}{m_{p l}}=\sqrt{\frac{r_{e}}{\alpha_{0}}}
$$

There is a dream,which,albeit more often not confessed,occupies the most secret aspirations of theoreticians and is that of reducing the various constants of Physics to simple formula involving integers and transcendent numbers. The fine-structure constant plays an important role in modern physics. Yet it continues to be a mystery as to exactly what it represents and why it has the mystical value it has. The purpose of many sciences is to find the most accurate mathematical formula that can be found in the experimental value of fine-structure constant. Attempts to find a mathematical basis for this dimensionless constant have continued up to the present time. However,no numerological explanation has ever been accepted by the physics community. We propose in [8] the exact formula for the fine-structure constant a with the golden angle,the relativity factor and the fifth power of the golden mean:

$$
a^{-1}=360 \cdot \varphi^{-2}-2 \cdot \varphi^{-3}+(3 \cdot \varphi)^{-5}
$$

with numerical value:

$$
a^{-1}=137,035999164 \ldots
$$

Another beautiful forms of the equations are:

$$
\begin{aligned}
& \frac{1}{\alpha}=\frac{360}{\varphi^{2}}-\frac{2}{\varphi^{3}}+\frac{1}{3^{5} \varphi^{5}} \\
& \frac{1}{\alpha}=\frac{360}{\varphi^{2}}-\frac{2}{\varphi^{3}}+\frac{3^{-5}}{\varphi^{5}}
\end{aligned}
$$

Other equivalent expressions for the fine-structure constant are:

$$
\begin{gathered}
a^{-1}=\left(362-3^{-4}\right) \cdot \varphi^{-2}-\left(1-3^{-5}\right) \cdot \varphi^{-1} \\
a^{-1}=\left(362-3^{-4}\right)+\left(3^{-4}+2 \cdot 3^{-5}-364\right) \cdot \varphi^{-1} \\
a^{-1}=1-2 \cdot \varphi^{-1}+360 \cdot \varphi^{-2}-\varphi^{-3}+(3 \cdot \varphi)^{-5}
\end{gathered}
$$




\section{Proton to electron mass ratio}

In Physics,the ratio of the mass of a proton to an electron is simply the remainder of the mass of the proton divided by that of the electron,from the system of units. Two of the great mysteries of physics are the origin of mass and the mysterious ratio of mass between a proton and an electron. The values of me and $\mathrm{mp}$,and the equilibrium between them,govern nuclear reactions such as the decay of protons and the nuclear synthesis of stars,leading to the formation of basic biochemical elements,including carbon. The space where stars and planets form and support life and molecular structures can appear. The mass ratio of protons to electrons, two constant particles that make up about $95 \%$ of the visible Universe,may be related to the total computational value of the Universe. Thus, as pure numbers they are supposed to be associated with prime numbers,entropy,binary and complexity.

The proton to electron mass ratio $\mu$ is a ratio of like-dimensioned physical quantities, it is a dimensionless quantity,a function of the dimensionless physical constants,and has numerical value independent of the system of units. Two of the great mysteries of physics are the origin of mass and the mysterious mass ratio between the proton and electron. The numerical challenge of the mass ratio of proton to electron in the field of elementary particle physics began with the discovery of the electron by JJ Thomson in 1.897, and with the identification of the point nature of the proton by $\mathrm{E}$. Rutherford in 1.911. These two particles have electric charges that are identical in size but opposite charges. The 2.018 CODATA recommended value of the proton to electron mass ratio is:

$$
\mu=1.836,15267343
$$

with standard uncertainty 0,00000011 and relative standard uncertainty $6,0 \times 10^{-11}$. The value of $\mu$ is a solution of the equation:

$$
3 \cdot \mu^{4}-5.508 \cdot \mu^{3}-841 \cdot \mu^{2}+10 \cdot \mu-2.111=0
$$

We propose in [9] the exact mathematical expression for the proton to electron mass ratio using Fibonacci and Lucas numbers:

$$
\mu^{32}=\varphi^{-42} \cdot F 5^{160} \cdot L^{47} \cdot L 19^{40 / 19}
$$

with numerical value:

$$
\mu=1.836,15267343 \ldots
$$

Also we propose in [9] the exact mathematical expression for the proton to electron mass ratio:

$$
\mu=165 \sqrt[3]{\frac{\ln ^{11} 10}{7}}
$$

with numerical value:

$$
\mu=1836,15267392 \ldots
$$

Other equivalent expressions for the proton to electron mass ratio are:

$$
\begin{gathered}
\mu^{3}=7^{-1} \cdot 165^{3} \cdot \ln ^{11} 10 \\
7 \cdot \mu^{3}=(3 \cdot 5 \cdot 11)^{3} \cdot \ln ^{11}(2 \cdot 5)
\end{gathered}
$$

Other exact mathematical expression in [9] for the proton to electron mass ratio is:

$$
\mu=6 \cdot \pi^{5}+\pi^{-3}+2 \cdot \pi^{-6}+2 \cdot \pi^{-8}+2 \cdot \pi^{-10}+2 \cdot \pi^{-13}+\pi^{-15}
$$


with numerical value:

$$
\mu=1.836,15267343 \ldots
$$

In [9] was presented the exact mathematical expressions that connect the proton to electron mass ratio $\mu$ and the fine-structure constant a:

$$
\begin{gathered}
9 \cdot \mu-119 \cdot a^{-1}=5 \cdot(\varphi+42) \\
\mu-6 \cdot a^{-1}=360 \cdot \varphi-165 \cdot \Pi+345 \cdot e+12 \\
\mu-182 \cdot a=141 \cdot \varphi+495 \cdot \Pi-66 \cdot e+231 \\
\mu-807 \cdot a=1.205 \cdot \Pi-518 \cdot \varphi-411 \cdot e
\end{gathered}
$$

\section{Gravitational coupling constant for the electron}

In physics, the gravitational coupling constant aG is a constant that characterizes the gravitational pull between a given pair of elementary particles. For the electron pair this constant is denoted by ag. The choice of units of measurement,but only with the choice of particles. The gravitational coupling constant a is a scaling ratio that can be used to compare similar unit values from different scaling systems (Planck scale,atomic scale, and cosmological scale). The gravitational coupling constant can be used for comparison of length,range and force values. The gravitational coupling constant ag is defined as:

$$
\mathrm{a}_{G}=\frac{G m_{e}^{2}}{\hbar c}
$$

There is so far no known way to measure ag directly. The value of the constant gravitational coupling ag is only known in four significant digits. The approximate value of the constant gravitational coupling ag is:

$$
\mathrm{aG}=1,7518099 \times 10^{-45}
$$

Also the gravitational coupling constant is universal scaling factor:

$$
\alpha_{G}=\frac{m_{e}^{2}}{m_{p l}^{2}}=\frac{\alpha_{G(p)}}{\mu^{2}}=\frac{\alpha}{\mu \mathrm{N}_{1}}=\frac{\alpha^{2}}{\mathrm{~N}_{1}^{2} \alpha_{G(p)}}=\left(\frac{2 \pi l_{p l}}{\lambda_{e}}\right)^{2}=\left(\alpha \frac{l_{p l}}{r_{e}}\right)^{2}=\left(\frac{l_{p l}}{\alpha_{0}}\right)^{2}
$$

\section{Gravitational coupling constant for the proton}

The gravitational coupling constant $\mathrm{aG}(\mathrm{p})$ for the proton is produced similar to the electron,but replaces the mass of electrons with the mass of the protons. The gravitational coupling constant of the proton $a \mathrm{G}(\mathrm{p})$ is defined as:

$$
a_{G(p)}=\frac{G m_{p}^{2}}{\hbar c}
$$

The approximate value of the constant gravitational coupling of the proton is:

$$
\mathrm{aG}(\mathrm{p})=5,9061512 \times 10^{-39}
$$

Also other expression for the gravitational coupling constant is: 


$$
\alpha_{G(p)}=\frac{m_{p}^{2}}{m_{p l}^{2}}=\mu^{2} \alpha_{G}=\frac{\alpha \mu}{\mathrm{N}_{1}}=\frac{\alpha^{2}}{\mathrm{~N}_{1}^{2} \alpha_{G}}
$$

\section{Ratio of electric force to gravitational force between electron and proton}

The enormous value of the ratio of electric force to gravitational force was first pointed out by Bergen Davis in 1.904. But Weyl and Eddington suggested that the number was about $10^{40}$ and was related to cosmological quantities. The electric force $F_{c}$ between electron and proton is defined as:

$$
F_{c}=\frac{q_{e}^{2}}{4 \pi \varepsilon_{0} r^{2}}
$$

The gravitational force $\mathrm{Fg}$ between electron and proton is defined as:

$$
F_{g}=\frac{G m_{e} m_{p}}{r^{2}}
$$

So from these expressions we have:

$$
\begin{gathered}
\mathrm{N}_{1}=\frac{F_{c}}{F_{g}} \\
\mathrm{~N}_{1}=\frac{q_{e}^{2}}{4 \pi \varepsilon_{0} G m_{e} m_{p}} \\
\mathrm{~N}_{1}=\frac{k_{e} q_{e}^{2}}{G m_{p} m_{e}} \\
\mathrm{~N}_{1}=\frac{\alpha \hbar c}{G m_{e} m_{p}}
\end{gathered}
$$

So the ratio $\mathrm{N} 1$ of electric force to gravitational force between electron and proton is defined as:

$$
\mathrm{N}_{1}=\frac{\alpha}{\mu \alpha_{G}}=\frac{\alpha \mu}{\alpha_{G(p)}}=\frac{a}{\sqrt{\alpha_{G} \alpha_{G(p)}}}=\frac{k_{e} q_{e}^{2}}{G m_{e} m_{p}}=\frac{\alpha \hbar c}{G m_{e} m_{p}}
$$

The approximate value of the ratio of electric force to gravitational force between electron and proton is:

$$
\mathrm{N}_{1}=2,26866072 \times 10^{39}
$$

The ratio $\mathrm{N} 1$ of electric force to gravitational force between electron and proton can also be written in expression:

$$
\mathrm{N}_{1}=\frac{5}{3} 2^{130}=2,26854911 \times 10^{39}
$$

According to current theories $\mathrm{N} 1$ should be constant. 


\section{Avogadro's number}

Avogadro's number NA is defined as the number of carbon-12 atoms in twelve grams of elemental carbon-12 in its standard state. Avogadro's number NA is the fundamental physical constant that links the macroscopic physical world of objects that we can see and feel with the submicroscopic,invisible world of atoms. The name honors the Italian mathematical physicist Amedeo Avogadro,who proposed that equal volumes of all gasses at the same temperature and pressure contain the same number of molecules. The most accurate definition of the Avogadro's number value involves the change in molecular quantities and,in particular,the change in the value of an elementary charge. The exact value of the Avogadro's number is:

$$
N A=6,02214076 \times 10^{23}
$$

The value of the Avogadro's number NA can also be written in expressions:

$$
\begin{gathered}
N A=84.446 .885^{3}=6,02214076 \times 10^{23} \\
N A=2^{79}=6,04462909 \times 10^{23}
\end{gathered}
$$

\section{New formula for the planck length}

A Planck length $\mid p l$ is about $10^{-20}$ times the diameter of a proton,meaning it is so small that immediate observation at this scale would be impossible in the near future. The length Planck Ipl has dimension [L]. The length Planck Ipl can be defined by three fundamental natural constants,the speed of light at vacuum c,the reduced Planck constant and the gravity constant $\mathrm{G}$ as:

$$
l_{p l}=\sqrt{\frac{\hbar G}{c^{3}}}=\frac{\hbar}{m_{p l} c}=\frac{h}{2 \pi m_{p l} c}=\frac{m_{p} r_{p}}{4 m_{p l}}
$$

The 2.018 CODATA recommended value of the Planck length is $\mid \mathrm{pl}=1,616255 \times 10^{-35} \mathrm{~m}$ with standard uncertainty $0,000018 \times 10^{-35} \mathrm{~m}$ and relative standard uncertainty $1,1 \times 10^{-5}$.

The Bohr radius ao is a physical constant, approximately equal to the most probable distance between the nucleus and the electron in a hydrogen atom in its ground state. The Bohr radius ao is defined as:

$$
\mathrm{a}_{0}=\frac{\hbar}{\alpha m_{e} c}=\frac{r_{e}}{\alpha^{2}}=\frac{\lambda_{c}}{2 \pi \alpha}
$$

The 2.018 CODATA recommended value of the Bohr radius is a0 $=5,29177210903 \times 10^{-11} \mathrm{~m}$ with standard uncertainty $0,00000000080 \times 10^{-11} \mathrm{~m}$ and relative standard uncertainty $1,5 \times 10^{-10}$.

The Planck constant,or Planck's constant,is a fundamental physical constant of foundational importance in quantum mechanics. The constant gives the relationship between the energy of a photon and its frequency,and by the mass-energy equivalence,the relationship between mass and frequency. Specifically,a photon's energy is equal to its frequency multiplied by the Planck constant. The constant is generally denoted by $\mathrm{h}$. The reduced Planck constant,equal to the constant divided by $2 \cdot \pi$, is denoted by $\hbar$. For the reduced Planck constant $\hbar$ apply:

$$
\hbar=a \cdot m e \cdot a 0 \cdot c
$$

So from these expressions we have:

$$
\begin{gathered}
\hbar^{2}=a^{2} \cdot m e^{2} \cdot a 0^{2} \cdot c^{2} \\
\left(\hbar \cdot G / c^{3}\right)=a^{2} \cdot m e^{2} \cdot a 0^{2} \cdot(G / \hbar \cdot c)
\end{gathered}
$$




$$
\begin{gathered}
\left(\hbar \cdot G / c^{3}\right)=a^{2} \cdot a 0^{2} \cdot\left(G \cdot m e^{2} / \hbar \cdot c\right) \\
|p|^{2}=a^{2} \cdot a G \cdot a 0^{2}
\end{gathered}
$$

So the new formula for the Planck length Ipl is:

$$
l_{p l}=a \sqrt{a_{G}} \mathrm{a}_{0}
$$

\section{New formula for the Avogadro's number}

Jeff Yee proposed in [10] that the mole and charge are related by deriving Avogadro's number from three constants, the Bohr radius, the Planck length and Euler's number. The fundamental unit of length in this unit system is the Planck length Ipl. Spacetime is proposed to be a lattice structure, in which its unit cells have sides of length a,marked below in the next figure. The lattice contains repeating cells with this structure,so it can be simplified to model a single unit cell of this repeating structure. These types of structures are commonly found in molecules. The center point of wave convergence is referred to here as a wave center. The separation length between granules in the unit cell is the diameter of a granule (2.|pl) multiplied by Euler's number (e), which is the base of the natural logarithm. There are exactly Avogadro's number of unit cells in the radius of hydrogen. The Avogadro's number NA can be calculated from the Planck length Ipl,the Bohr radius ao and Euler's number e:

$$
\mathrm{N}_{\mathrm{A}}=\frac{\mathrm{a}_{0}}{2 e l_{p l}}
$$

We will use this expression and the new formula for the Planck length Ipl to resulting the unity formula that connects the fine-structure constant $\mathrm{a}$ and the gravitational coupling constant aG:

$$
\begin{gathered}
\mathrm{a} 0=2 \cdot \mathrm{e} \cdot \mathrm{NA} \cdot \mathrm{Ipl} \\
\mathrm{a}_{0}=2 e \mathrm{~N}_{\mathrm{A}} a \sqrt{\mathrm{a}_{G}} \mathrm{a}_{0} \\
2 e \mathrm{~N}_{\mathrm{A}} a \sqrt{\mathrm{a}_{G}}=1
\end{gathered}
$$

Therefore the unity formula that connect the fine-structure constant a,the gravitational coupling constant aG and the Avogadro's number NA is:

$$
4 \cdot e^{2} \cdot a^{2} \cdot a G \cdot N A^{2}=1
$$

The unity formula is equally valid:

$$
a^{2} \cdot a G=(2 \cdot e \cdot N A)^{-2}
$$

This formula is the simple unification of the electromagnetic and the gravitational interactions. So from this expression the new formula for the Avogadro number NA is:

$$
\mathrm{N}_{\mathrm{A}}=\left(2 e a \sqrt{\mathrm{a}_{G}}\right)^{-1}
$$

\section{Mathematical formulas that connect 3 dimensionless physical constants}

The exact mathematical formula that connect the mass ratio of proton to electron,the fine-structure constant a and the 
proton-proton gravitational coupling constant $\mathrm{aG}(\mathrm{pp})$ is:

$$
a^{7}=\mu^{7} \cdot[a G(p p) \cdot \log 2(2 \cdot \pi)]
$$

The exact mathematical formula that connect the mass ratio of proton to electron,the fine-structure constant a and the proton-electron gravitational coupling constant $\mathrm{aG}(\mathrm{pe})$ is:

$$
a^{7}=\mu^{8} \cdot[a G(p p) \cdot \log 2(2 \cdot \pi)]
$$

The exact mathematical formula that connect the mass ratio of proton to electron,the fine-structure constant a and the gravitational coupling constant of electrons-electrons aG(ee) is:

$$
a^{7}=\mu^{9} \cdot\left[a G(p p) \cdot \log _{2}(2 \cdot n)\right]
$$

The exact mathematical formula that connect the proton to electron mass ratio $\mu$, the gravitational coupling constant aG of the electron and the gravitational coupling constant of the proton $\mathrm{aG}(\mathrm{p})$ is:

$$
a G(p)=\mu^{2} \cdot a G
$$

The exact mathematical formula that connect the fine-structure constant a,the gravitational coupling constant ag of the electron and the Avogadro number NA is:

$$
4 \cdot e^{2} \cdot a^{2} \cdot a G \cdot N A^{2}=1
$$

\section{Mathematical formulas that connect 4 dimensionless physical constants}

The exact mathematical formula that connect the proton to electron mass ratio $\mu$,the fine-structure constant $\mathrm{a}$, the ratio $\mathrm{N} 1$ of electric force to gravitational force between electron and proton and the gravitational coupling constant ag of the electron is:

$$
a=\mu \cdot N_{1} \cdot a G
$$

The exact mathematical formula that connect the proton to electron mass ratio $\mu$, the fine-structure constant $\mathrm{a}$,the ratio $\mathrm{N} 1$ of the electric force to the gravitational force between electron and proton and the gravitational coupling constant of the proton $\mathrm{aG}(\mathrm{p})$ is:

$$
\mathrm{a} \cdot \mu=\mathrm{N}_{1} \cdot \mathrm{aG}(\mathrm{p})
$$

The exact mathematical formula that connect the fine-structure constant a,the ratio $\mathrm{N} 1$ of the electric force to the gravitational force between electron and proton,the gravitational coupling constant aG of the electron and the gravitational coupling constant of the proton $\mathrm{aG}(\mathrm{p})$ is:

$$
\mathrm{a}^{2}=\mathrm{N} 1^{2} \cdot \mathrm{aG} \cdot \mathrm{aG}(\mathrm{p})
$$

The exact mathematical formula that connect the proton to electron mass ratio $\mu$,the fine-structure constant a,the Avogadro's number NA and the gravitational coupling constant of the proton $a G(p)$ is:

$$
\mu^{2}=4 \cdot e^{2} \cdot a^{2} \cdot a G(p) \cdot N A^{2}
$$

The exact mathematical formula that connect the proton to electron mass ratio $\mu$, the fine-structure constant a,the ratio $\mathrm{N}_{1}$ of the electric force to the gravitational force between electron and proton and the Avogadro's number NA is: 


$$
\mu \cdot N 1=4 \cdot e^{2} \cdot a^{3} \cdot N A^{2}
$$

\section{Mathematical formulas that connect 5 dimensionless physical constants}

The exact mathematical formula that connect the proton to electron mass ratio $\mu$,the fine-structure constant a,the ratio $\mathrm{N} 1$ of the electric force to the gravitational force between electron and proton,the Avogadro's number NA and the gravitational coupling constant aG of the electron is:

$$
4 \cdot e^{2} \cdot a \cdot \mu \cdot a G^{2} \cdot N A^{2} \cdot N 1=1
$$

The exact mathematical formula that connect the proton to electron mass ratio $\mu$,the fine-structure constant $\alpha$, the ratio $\mathrm{N} 1$ of the electric force to the gravitational force between electron and proton,the Avogadro's number NA and the gravitational coupling constant of the the proton $\mathrm{aG}(\mathrm{p})$ is:

$$
\mu^{3}=4 \cdot e^{2} \cdot a \cdot a G(p)^{2} \cdot N A^{2} \cdot N 1
$$

The exact mathematical formula that connect the proton to electron mass ratio $\mu$,the ratio $N 1$ of the electric force to the gravitational force between electron and proton,the Avogadro's number NA,the gravitational coupling constant aG of the electron and the gravitational coupling constant of the proton $\mathrm{aG}(\mathrm{p})$ is:

$$
\mu^{2}=4 \cdot e^{2} \cdot a G \cdot a G(p)^{2} \cdot N A^{2} \cdot N 1^{2}
$$

\section{Mathematical formulas that connect 6 dimensionless physical constants}

The exact mathematical formula that connect the proton to electron mass ratio $\mu$,the fine-structure constant $a$, the ratio $\mathrm{N} 1$ of electric force to gravitational force between electron and proton, the Avogadro's number NA,the gravitational coupling constant aG of the electron and the gravitational coupling constant of the proton $\mathrm{aG}(\mathrm{p})$ is:

$$
\mu=4 \cdot e^{2} \cdot a \cdot a G \cdot a G(p) \cdot N A^{2} \cdot N 1
$$

\section{Gravitational Constant}

The gravitational constant is an empirical physical constant that participates in the calculation of gravitational force between two bodies and is denoted by the letter G. It usually appears in Isaac Newton's law of universal gravitation and Albert Einstein's general theory of relativity. The physicist Sir Isaac Newton in 1.687 published his book "Philosophiae Naturalis Principia Mathematica" where he presented the law of universal gravity to describe and calculate the mutual attraction of particles and huge objects in the universe. In this paper,Isaac Newton concluded that the attraction between two bodies is proportional to the product of their masses and inversely proportional to the square of the distance separating them. However,these must be adjusted by introducing the gravity constant $\mathrm{G}$. The gravitational constant $\mathrm{G}$ occupies an anomalous position among the other constants of physics. The mass $M$ of any celestial object cannot be determined independently of the gravitational attraction that it exerts. Thus, the combination $G \cdot M$, not the separate value of $M$,is the only meaningful property of a star,planet,or galaxy. According to general relativity and the principle of equivalence, $G$ does not depend on material properties but is in a sense a geometric factor. The gravitational constant is defined as:

$$
G=\alpha_{G} \frac{\hbar c}{m_{e}^{2}}
$$

The 2.018 CODATA recommended value of gravitational constant $G=6,67430 \times 10^{-11} \mathrm{~m} / \mathrm{kg} \cdot \mathrm{s}^{2}$ with standard uncertainty $0,00015 \times 10^{-11} \mathrm{~m}^{3} / \mathrm{kg} \cdot \mathrm{s}^{2}$ and relative standard uncertainty $2,2 \times 10^{-5}$. Now we will find the formula for the gravitational constant $\mathrm{G}$ using the unity formulas that we calculated. From expression (13) the gravitational coupling constant aG can be written in the form: 


$$
\begin{aligned}
& 4 \cdot e^{2} \cdot N A^{2} \cdot a^{2} \cdot a G=1 \\
& a G=(2 \cdot e \cdot a \cdot N A)^{-2}
\end{aligned}
$$

Therefore from this expression the formula for the gravitational constant is:

$$
G=\left(2 e a \mathrm{~N}_{\mathrm{A}}\right)^{-2} \frac{\hbar c}{m_{e}^{2}}
$$

\section{Cosmological constant}

The relevant constant in atomic physics is the fine-structure constant a,which plays a fundamental role in atomic physics and quantum electrodynamics. The analogous constant in cosmology is the gravitational fine-structure constant ag. It plays a fundamental role in cosmology. The mysterious value of the gravitational fine-structure constant ag is an equivalent way to express the biggest issue in theoretical physics. The new formula for the Planck length Ipl is:

$$
l_{p l}=a \sqrt{a_{G}} \mathrm{a}_{0}
$$

The fine-structure constant equals:

$$
\alpha^{2}=\frac{r_{e}}{a_{0}}
$$

From these expressions we have:

$$
\begin{gathered}
l_{p l}=\frac{a \sqrt{\alpha_{G}} r_{e}}{\alpha^{2}} \\
l_{p l}=\frac{\sqrt{\alpha_{G}}}{a} r_{e} \\
\frac{l_{p l}^{3}}{r_{e}^{3}}=\frac{\sqrt{\alpha_{G}^{3}}}{a^{3}}
\end{gathered}
$$

The gravitational fine structure constant ag is defined as:

$$
\begin{gathered}
\alpha_{g}=\frac{l_{p l}^{3}}{r_{e}^{3}} \\
\alpha_{g}=\frac{\sqrt{\alpha_{G}^{3}}}{\alpha^{3}} \\
\alpha_{g}=\sqrt{\frac{\alpha_{G}^{3}}{\alpha^{6}}}
\end{gathered}
$$


with numerical value:

$$
a g=1,886837 \times 10^{-61}
$$

Also equals:

$$
\begin{aligned}
& a g^{2} \cdot a^{6}=a g^{3} \\
& a g^{2}=a G^{3} \cdot a^{-6} \\
& a_{g}^{2}=\left(\frac{a_{G}}{a^{2}}\right)^{3}
\end{aligned}
$$

From the expressions (13) and (28) resulting the formula for the gravitational fine-structure constant ag:

$$
a g=\left(2 \cdot e \cdot a^{2} \cdot N A\right)^{-3}
$$

Also apply the expressions:

$$
\begin{aligned}
& \left(2 \cdot e \cdot a^{2} \cdot N A\right)^{3} \cdot a g=1 \\
& 8 \cdot e^{3} \cdot a^{6} \cdot a g \cdot N A^{3}=1
\end{aligned}
$$

In the context of cosmology the cosmological constant is a homogeneous energy density that causes the expansion of the universe to accelerate. Originally proposed early in the development of general relativity in order to allow a static universe solution it was subsequently abandoned when the universe was found to be expanding. Now the cosmological constant is invoked to explain the observed acceleration of the expansion of the universe. The cosmological constant is the simplest realization of dark energy,which is the more generic name given to the unknown cause of the acceleration of the universe. Its existence is also predicted by quantum physics, where it enters as a form of vacuum energy,although the magnitude predicted by quantum theory does not match that observed in cosmology.

The cosmological constant $\Lambda$ is presumably an enigmatic form of matter or energy that acts in opposition to gravity and is considered by many physicists to be equivalent to dark energy. Nobody really knows what the cosmological constant is exactly,but it is required in cosmological equations in order to reconcile theory with our observations of the universe. One potential explanation for the cosmological constant lies in the realm of modern particle physics. Experiments have verified that empty space is permeated by countless virtual particles constantly popping in and out of existence. It is commonly believed that the cosmological constant problem can only be solved ultimately in a unified theory of quantum gravity and the standard model of electroweak and strong interactions, which is still absent so far. But connecting vacuum energy to the cosmological constant is not straightforward. Based on their observations of supernovas, astronomers estimate that dark energy should have a small and sedate value,just enough to push everything in the universe apart over billions of years. Yet when scientists try to calculate the amount of energy that should arise from virtual particle motion,they come up with a result that's 120 orders of magnitude greater than what the supernova data suggest. The cosmological constant has the same effect as an intrinsic energy density of the vacuum,pvac and an associated pressure. In this context, it is commonly moved onto the right-hand side of the equation, and defined with a proportionality factor of $\Lambda=8 \cdot \Pi \cdot \rho$ vac where unit conventions of general relativity are used (otherwise factors of $\mathrm{G}$ and c would also appear,i.e:

$$
\Lambda=8 \pi \rho_{v a c} \frac{G}{c^{4}}=\kappa \rho_{v a c}
$$

where $\mathrm{k}$ is Einstein's rescaled version of the gravitational constant $\mathrm{G}$. The cosmological constant has been introduced in gravitational field equations by Einstein in 1.917 in order to satisfy Mach's principle of the relativity of inertia. Then it was demonstrated by Cartan in 1.922 that the Einstein field tensor including a cosmological constant $\Lambda$ : 


$$
\mathrm{E}_{\mu v}=R_{\mu v}-\frac{1}{2} R g_{\mu v}+\Lambda g_{\mu v}
$$

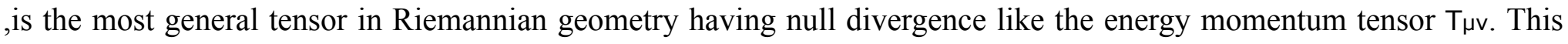
theorem has set the general form of Einstein's gravitational field equations as $E_{\mu v}=\kappa \cdot T_{\mu v}$ and established from first principles the existence of $\Lambda$ as an unvarying true constant. The cosmological constant problem dates back to the realization that it is equivalent to a vacuum energy density. One of the main consequences in cosmology of a positive cosmological constant is an acceleration of the expansion of the universe. Such an acceleration has been first detected in 1.981 in the Hubble diagram of infrared elliptical galaxies,yielding a positive value close to the presently measured one,but with still large uncertainties. Accurate measurements of the acceleration of the expansion since 20 years have reinforced the problem. The cosmological constant $\Lambda$,as it appears in Einstein's equations, is a curvature. As such,besides being an energy density, it is also the inverse of the square of an invariant cosmic length $L$.

In the early-mid 20th century Dirac and Zel'dovich were among the first scientists to suggest an intimate connection between cosmology and atomic physics. Though a revolutionary proposal for its time,Dirac's Large Number Hypothesis (1.937) adopted a standard assumption of the non-existence of the cosmological constant term $\Lambda=0$. Zel'dovich insight (1.968) was to realize that a small but nonzero cosmological term $\Lambda>0$ allowed the present day radius of the Universe to be identified with the de Sitter radius which removed the need for time dependence in the fundamental couplings. Thus, he obtained the formula:

$$
\Lambda=\frac{m_{p}^{6} G^{2}}{\hbar^{6}}
$$

where $\mathrm{m}$ is a mass scale characterizing the relative strengths of the gravitational and electromagnetic interactions, which he identified with the proton mass $\mathrm{mp}$.

Laurent Nottale in [11] which,instead,suggests the identification $m=m e / a$. He assumed that the cosmological constant $\wedge$ is the sum of a general-relativistic term and of the quantum,scale-varying,gravitational self-energy of virtual pairs. A renormalization group approach is used to describe its scale-dependence. We argue that the large scale value of $\wedge$ is reached at the classical electron scale. This reasoning provides with a large-number relation:

$$
\alpha \frac{m_{p l}}{m_{e}}=\left(\frac{L}{l_{p l}}\right)^{\frac{1}{3}}
$$

The cosmological constant $\Lambda$ has the dimension of an inverse length squared. The cosmological constant is the inverse of the square of a length $L$ :

$$
L=\sqrt{\Lambda^{-1}}
$$

For the de Sitter radius equals:

$$
R_{d}=\sqrt{3} L
$$

So the de Sitter radius and the cosmological constant are related through a simple equation:

$$
R_{d}=\sqrt{\frac{3}{\Lambda}}
$$

From this equation resulting the expressions for the gravitational fine structure constant ag:

$$
\alpha \frac{m_{p l}}{m_{e}}=\left(l_{p l} \sqrt{\Lambda}\right)^{-\frac{1}{3}}
$$




$$
\begin{gathered}
\alpha_{g}=l_{p l} \sqrt{\Lambda} \\
\alpha_{g}=\sqrt{\frac{G \hbar \Lambda}{c^{3}}}
\end{gathered}
$$

So the cosmological constant $\Lambda$ equals:

$$
\begin{gathered}
\Lambda=\alpha_{g}^{2} l_{p l}^{-2} \\
\Lambda=\frac{l_{p l}^{4}}{r_{e}^{6}} \\
\Lambda=\alpha_{g}^{2} \frac{c^{3}}{G \hbar} \\
\Lambda=\frac{G}{\hbar^{4}}\left(\frac{m_{e}}{a}\right)^{6}
\end{gathered}
$$

From the expression (28) resulting the simple unification of atomic physics and cosmology:

$$
\begin{gathered}
a g=\left(2 \cdot e \cdot a^{2} \cdot N A\right)^{-3} \\
|p|^{2} \cdot \Lambda=\left(2 \cdot e \cdot a^{2} \cdot N A\right)^{-6} \\
\left(2 \cdot e \cdot a^{2} \cdot N A\right)^{6} \cdot|p|^{2} \cdot \Lambda=1
\end{gathered}
$$

Now we will use the unity formula of the simple unification of atomic physics and cosmology to find the equation of the cosmological constant. For the cosmological constant equals:

$$
\Lambda=\left(2 e \alpha^{2} \mathrm{~N}_{\mathrm{A}}\right)^{-6} \frac{c^{3}}{G \hbar}
$$

\section{Conclusions}

We presented new exact formula for the fine-structure constant $a$ in terms of the golden angle,the relativity factor and the fifth power of the golden mean:

$$
a^{-1}=360 \cdot \varphi^{-2}-2 \cdot \varphi^{-3}+(3 \cdot \varphi)^{-5}
$$

A new interpretation and a very accurate value of the fine-structure constant has been discovered in terms of the golden radio.

We propose the exact equivalent mathematical expression for the proton to electron mass ratio using Fibonacci and Lucas numbers:

$$
\mu^{32}=\varphi^{-42} \cdot F 5^{160} \cdot L 5^{47} \cdot L 19^{40 / 19}
$$


We propose the exact mathematical expressions for the proton to electron mass ratio:

$$
\mu^{3}=7^{-1} \cdot 165^{3} \cdot \ln ^{11} 10
$$

Also other exact mathematical expression in for the proton to electron mass ratio is:

$$
\mu=6 \cdot \pi^{5}+\pi^{-3}+2 \cdot \pi^{-6}+2 \cdot \pi^{-8}+2 \cdot \pi^{-10}+2 \cdot \pi^{-13}+\pi^{-15}
$$

We present the exact mathematical expressions that connect the proton to electron mass ratio and the fine-structure constant:

$$
\begin{gathered}
9 \cdot \mu-119 \cdot a^{-1}=5 \cdot(\varphi+42) \\
\mu-6 \cdot a^{-1}=360 \cdot \varphi-165 \cdot \Pi+345 \cdot e+12 \\
\mu-182 \cdot a=141 \cdot \varphi+495 \cdot n-66 \cdot e+231 \\
\mu-807 \cdot a=1.205 \cdot \Pi-518 \cdot \varphi-411 \cdot e
\end{gathered}
$$

The new formula for the Planck length Ipl is:

$$
l_{p l}=a \sqrt{a_{G}} \mathrm{a}_{0}
$$

The new formula for the Avogadro's number NA is:

$$
\mathrm{N}_{\mathrm{A}}=\left(2 e a \sqrt{\alpha_{G}}\right)^{-1}
$$

It was presented the formula of the simple unification of the electromagnetic and the gravitational interactions:

$$
a^{2} \cdot a G=(2 \cdot e \cdot N A)^{-2}
$$

The mathematical formulas that connect dimensionless physical constants are:

$$
\begin{gathered}
a G(p)=\mu^{2} \cdot a G \\
a=\mu \cdot N 1 \cdot a G \\
a \cdot \mu=N 1 \cdot a G(p) \\
a^{2}=N 1^{2} \cdot a G \cdot a G(p) \\
4 \cdot e^{2} \cdot a^{2} \cdot a G \cdot N A^{2}=1 \\
\mu^{2}=4 \cdot e^{2} \cdot a^{2} \cdot a G(p) \cdot N A^{2} \\
\mu \cdot N 1=4 \cdot e^{2} \cdot a^{3} \cdot N A^{2} \\
4 \cdot e^{2} \cdot a \cdot \mu \cdot a G^{2} \cdot N A^{2} \cdot N 1=1 \\
\mu^{3}=4 \cdot e^{2} \cdot a \cdot a G(p)^{2} \cdot N A^{2} \cdot N 1 \\
\mu^{2}=4 \cdot e^{2} \cdot a G \cdot a G(p)^{2} \cdot N A^{2} \cdot N 1^{2} \\
\mu=4 \cdot e^{2} \cdot a \cdot a G \cdot a G(p) \cdot N A^{2} \cdot N 1
\end{gathered}
$$


The new formula for gravitational constant $\mathrm{G}$ is:

$$
G=\left(2 e a \mathrm{~N}_{\mathrm{A}}\right)^{-2} \frac{\hbar c}{m_{e}^{2}}
$$

The simple unification of atomic physics and cosmology is:

$$
|p|^{2} \cdot \Lambda=\left(2 \cdot e \cdot a^{2} \cdot N A\right)^{-6}
$$

Finally the formula for the cosmological constant is:

$$
\Lambda=\left(2 e \mathrm{a}^{2} \mathrm{~N}_{\mathrm{A}}\right)^{-6} \frac{c^{3}}{G \hbar}
$$

All these equations are simple,elegant and symmetrical in a great physical meaning.

\section{References}

[1] www.en.wikipedia.org/

[2] www.wolframalpha.com/

[3] www.math.stackexchange.com/

[4] www.mathworld.wolfram.com/

[5] www.numberempire.com/

[6] http://physics.nist.gov/cuu/Constants/

[7] Stergios Pellis Unification Archimedes constant $\pi$,golden ratio $\varphi$,Euler's Number e and imaginary number $i$ http://dx.doi.org/10.2139/ssrn.3975869, 2.021

[8] Stergios Pellis Exact formula for the fine-structure constant $\alpha$ in terms of the golden ratio $\varphi$ DOI: $\underline{10.13140 / R G .2 .2 .34473 .26728}, 2.021$

[9] Stergios Pellis Exact mathematical expressions of the proton to electron mass ratio with symmetrical shape and physical meaning http://dx.doi.org/10.2139/ssrn.3967998, 2.021

[10] Jeff Yee The Relationship of the Mole and Charge. https://vixra.org/pdf/1904.0445v1.pdf, 2.019

[11] L. Nottale, Mach's principle,Dirac's large numbers and the cosmological constant problem http://www.luth.obspm.fr/\%7Eluthier/nottale/arlambda.pdf, 1.993 